\title{
NEW EXAMPLES OF $p$-ADICALLY RIGID AUTOMORPHIC FORMS
}

\author{
JoËL BELLAÏCHE
}

\begin{abstract}
In this paper, we prove two $p$-adic rigidity results for automorphic forms for the quasi-split unitary group in three variables $\mathrm{U}(2,1)$ attached to a quadratic imaginary field.

We show first that the discrete automorphic forms for this group that are cohomological in degree 1 (and refined, with a non semi-ordinary refinement) are rigid, in the sense that they can not be interpolated in a positive dimensional $p$-adic family, even though the set of Hodge-Tate weights of all such forms is not $p$-adically discrete. This results implies that the eigenvariety of $\mathrm{U}(2,1)$ in cohomological degree 1 , if it exists in the sense of $[\mathrm{E}]$ (or [BC2]), is not equi-dimensional. Hence the situation for the quasisplit unitary group is in striking contrast with the one for its definite inner form $\mathrm{U}(3)$ and more generally any definite reductive group.

We then show that some of the automorphic forms considered above, namely the ones that are minimally ramified in their $A$-packet and attached to an Hecke character whose $L$-function does not vanish at the center of its functional equation, are even rigid in the stronger sense that they can not be put in a non trivial family interpolating cohomological automorphic forms in any degree.
\end{abstract}

\section{Acknowledgements}

This paper arose from a question that Laurent Clozel asked me in 1997, when I was a young graduate student under his supervision. The question was in substance: "the representation $\prod_{v \text { place of } \mathbb{Q}} \pi^{n}\left(\chi_{0}\right)_{v}$ is cuspidal for $\mathrm{U}(2,1)$ if and only if $L\left(s, \chi_{0}\right)$ vanishes at $s=1 / 2$ at an even order. Is it possible to infer some arithmetically significant informations from this fact?". Let this be the occasion to thank Laurent Clozel for this question, and also for all his help during my $\mathrm{PhD}$ and thereafter. I also thank Gaëtan Chenevier, with which I had innumerable conversations on subjects related to this article, for his patience, encouragement, and help. Finally, I'd like to thank Ken Tignor for many nice math talks, some of them related to this article. During the writing of this article, I was supported by the NSF grants DMS 05-01023 and DMS 08-01205.

\section{Introduction}

We denote by $\mathrm{U}(2,1)$ the quasi-split unitary group in three variables attached to a quadratic imaginary field, and we fix a prime $p$ at which $\mathrm{U}(2,1)$ splits.

The main aim of this note is to show (Theorem 1 ) that automorphic representations for $\mathrm{U}(2,1)$ that are cohomological in degree 1 , together with a suitable choice of a

Received by the editors July 16, 2009.

2000 Mathematics Subject Classification. 11F33. Secondarily: 11F80, 11 F67. 
refinement ${ }^{1}$ at $p$ are $p$-adically rigid, i.e. cannot be put in any non-trivial positivedimensional $p$-adic family.

This result is a little bit surprising, at least for me, since, as we shall see, the set of weights of those automorphic representations is not $p$-adically discrete, and is in fact dense in a 1-dimensional ${ }^{2}$ subspace of the weight space. Furthermore, the same automorphic representations with another choice of refinement can easily be put in a non-trivial 1-dimensional $p$-adic family (see Remark 4 ). Hence the eigenvariety for $\mathrm{U}(2,1)$ and cohomological degree 1 , if it exists in any reasonable sense (e.g [E]), is not equidimensional. Even more, different refinements (or $p$-stabilizations) of the same automorphic form may lie in components of different dimensions of the eigenvariety.

A rigidity result for some examples of ordinary, non essentially self-dual cohomological automorphic forms of $\mathrm{GL}_{3}$ has already been obtained by Ash, Pollack, and Stevens [APS]. They also conjecture that all non essentially self-dual automorphic forms for $\mathrm{GL}_{n}, n \leq 3$ are rigid. There is no doubt that their result is much deeper, both in significance and in difficulty, but the point of this paper is to show that a different kind of pathologies may occur in the case of unitary groups.

Non self-dual cohomological automorphic forms for $\mathrm{GL}_{n}$ are very mysterious. If $n \geq 3$, there is no Shimura varieties for $\mathrm{GL}_{n}$ or any of its inner form, and no one knows how to attach a Galois representation to a non self-dual cohomological automorphic forms for $\mathrm{GL}_{n}$. Similarly, $\mathrm{GL}_{n}(\mathbb{R})$ has no discrete series for $n \geq 3$ and no one ever constructed a non-trivial, positive-dimensional families for those groups except by using functoriality from some other groups. The rigidity result of [APS] for some automorphic forms for $\mathrm{GL}_{3}$ fits well in this picture.

The case of $\mathrm{U}(2,1)$ is very different. Hida constructed nice, equidimensional (of the expected dimension) families in the ordinary case for this group and many other a long time ago, and we also have such families for the definite inner form $\mathrm{U}(3)$ of $\mathrm{U}(2,1)$ (and higher rank analogs) without the ordinarity assumption, by works of Chenevier $[\mathrm{C}]$ and Emerton [E]. Urban and Skinner claim in [SU] that they are able to construct such families in the semi-ordinary case, at least for representations that are holomorphic discrete series at infinity. This note shows that in the non-definite, non ordinary case, pathologies may occur, and that beyond a general theorem on the existence of eigenvarieties (like, e.g. [E]), a formula computing the dimension of their components containing a refined automorphic representation would be very useful, and that this formula should depend not only on the given representation, but also on the chosen refinement.

The discussion above was essentially concerned with families of automorphic forms that are cohomological in a fixed degree - as in the works of Stevens, Chenevier, Emerton, and others. Our second result (Theorem 2) deals with families where forms are cohomological, but not necessarily of the same degree. Our result is that through some very specific (but arithmetically interesting) examples of non-tempered representations of $\mathrm{U}(2,1)$, even such more general families do not exist (more precisely, have dimension 0. )

\footnotetext{
${ }^{1}$ Some authors, like [SU], call the choice of a refinement a $p$-stabilization.

${ }^{2}$ In all this introduction, we are ruling out central (that is, anticyclotomic) deformations. Otherwise, the dimension would be 2 .
} 


\section{Preliminaries}

2.1. Unitary groups. Let $E$ be a quadratic imaginary field, and let $G=\mathrm{U}(2,1)$ be the unique quasi-split unitary group in three variables over $\mathbb{Q}$ that splits in $E$.

Let $p$ be a prime number that, we shall assume, splits in $E$.

Let $K^{p}=\prod_{l \neq p} K_{l}$ be a compact open subgroup of $G\left(\mathbb{A}_{f}^{p}\right)$ that we will call the tame level. We denote by $S$ the finite set of primes $l$ such that either $l=p$, or $l \neq p$ and $K_{l}$ is not maximal hyperspecial. For $l \notin S$, we denote by $\mathcal{H}_{l}$ the Hecke algebra (over $\mathbb{Z})$ of $G\left(\mathbb{Q}_{l}\right)$ with respect to $K_{l}$. We denote by $K_{p}$ a maximal compact hyperspecial subgroup of $G\left(\mathbb{Q}_{p}\right)$, by $I_{p}$ an Iwahori subgroup of $K_{p}$ and by $\mathcal{A}_{p}$ the Atkin-Lehner sub-algebra of the Hecke algebra of $G\left(\mathbb{Q}_{p}\right)$ with respect to $I_{p}$ - See $[\mathrm{BC} 2, \S 6.4 .4]$. We set $\mathcal{H}=\left(\otimes_{l \notin S} \mathcal{H}_{l}\right) \otimes \mathcal{A}_{p}$. This is a commutative algebra over $\mathbb{Z}$.

2.2. Automorphic refinements. We refer to $[\mathrm{BC} 2, \S 5.4]$ for a complete discussion of the notion of refinement $\mathcal{R}$ of an unramified representation $\pi_{p}$ of $G\left(\mathbb{Q}_{p}\right)$. Let us simply define it here as a character $\psi_{\mathcal{R}}: \mathcal{A}_{p} \rightarrow \mathbb{C}$ appearing in the $\mathcal{A}_{p}$-module $\pi_{p}^{I_{p}}$.

2.3. Hecke characters. Set $K=K_{p} \times K^{p}$. A refined representation $(\pi, \mathcal{R})$ of level $K$ shall mean a discrete automorphic representation $\pi$ for $\mathrm{U}(2,1)$, such that $\pi^{K} \neq 0$, together with a refinement $\mathcal{R}$ of $\pi_{p}$. Such a refined representation defines a character $\psi_{\pi, \mathcal{R}}=\psi_{\pi} \otimes \psi_{\mathcal{R}}: \mathcal{H} \rightarrow \mathbb{C}$. Here, $\psi_{\pi}:\left(\bigotimes_{l \notin S} \mathcal{H}_{l}\right) \rightarrow \mathbb{C}$ is the character giving the action of $\left(\bigotimes_{l \notin S} \mathcal{H}_{l}\right)$ on the one-dimensional space $\prod_{l \notin S} \pi_{l}^{K_{l}}$, and $\psi_{\mathcal{R}}$ is the character of $\mathcal{A}_{p}$ defined by the refinement $\mathcal{R}$.

If $\pi_{\infty}$ is cohomological (for some coefficient system), the image of $\psi_{\pi, \mathcal{R}}$ lies in fact in a number field. We fix once and for all an embedding $\overline{\mathbb{Q}} \subset \overline{\mathbb{C}}$, and also an embedding $\overline{\mathbb{Q}} \subset \overline{\mathbb{Q}}_{p}$. Hence we can see $\psi_{\pi, \mathcal{R}}$ as a character $\mathcal{H} \rightarrow \overline{\mathbb{Q}}_{p}$ when $\pi_{\infty}$ is cohomological. Conversely, we shall say that a character $\psi_{0}: \mathcal{H} \rightarrow \overline{\mathbb{Q}}_{p}$ is of level $K$ and cohomological of degree $n$ if it is of the form $\psi_{\pi, \mathcal{R}}$ for $(\pi, \mathcal{R})$ a refined representation of level $K$ such that $\pi_{\infty}$ is cohomological in degree $n$.

2.4. Galois representations. To a discrete automorphic representation $\pi$ such that $\pi_{\infty}$ is cohomological of some degree is attached by the work of Blasius and Rogawski ([BR1, Thm 1.9.1]) and the whole book [ZFPMS] a three dimensional representation (see $[\mathrm{BC} 1, \S 3.2 .2]$ for this formulation of the results of Blasius and Rogawski) $\rho_{\pi}$ : $\Gamma_{E} \rightarrow \mathrm{GL}_{3}\left(\overline{\mathbb{Q}}_{p}\right)$ that depends only of the Hecke-character $\psi_{\pi}$ (here and below, $\Gamma_{F}$ denotes "the" absolute Galois group of a field $F$ )

The representation $\rho_{\pi}$ satisfies the symmetry condition $\rho_{\pi} \simeq \rho_{\pi}^{\perp}$, where for $\rho$ a representation of $\Gamma_{E}, \rho^{\perp}(g)={ }^{t} \rho(c g c)^{-1}$. Here, $c$ is an element of $\Gamma_{\mathbb{Q}}-\Gamma_{E}$ of order 2 .

We fix once and for all a place $v$ of $E$ above $p$. The decomposition group $D_{v}$ at $v$ in $\Gamma_{E}$ is isomorphic to $\operatorname{Gal}\left(\overline{\mathbb{Q}}_{p} / \mathbb{Q}_{p}\right)$. The choice of $v$ determines an isomorphism $G\left(\mathbb{Q}_{p}\right) \simeq \mathrm{GL}_{3}\left(\mathbb{Q}_{p}\right)$, well-defined up to conjugation.

If $\pi_{p}$ is unramified, then $\rho_{\pi} \mid D_{v}$ is crystalline. It thus has three integral HodgeTate weights, that we will denote by $k_{0} \leq k_{1} \leq k_{2}$ and three Frobenius eigenvalues $\phi_{0}, \phi_{1}, \phi_{2}$. The weak admissibility condition of crystalline representation theory implies the equality $v_{p}\left(\phi_{0}\right)+v_{p}\left(\phi_{1}\right)+v_{p}\left(\phi_{2}\right)=k_{0}+k_{1}+k_{2}$, where $v_{p}$ is the $p$-adic valuation. 
We will refer to the $k_{i}$ 's and the $\phi_{i}$ 's simply as the weights and eigenvalues of $\rho_{\pi}$, without mentioning the choice of the place $v$ above $p$.

2.5. Refinement on the Galois side. For our purposes, a refinement of $\rho_{\pi}$ is simply an ordering of the eigenvalues $\phi_{0}, \phi_{1}, \phi_{2}$. In a generic situation, there is thus 6 refinements, and less if two eigenvalues are equal. The important fact for us (see $[\mathrm{BC} 1, \S 6])$ is that each automorphic refinement at $p$ of $\pi$ defines canonically a Galois refinement of $\rho_{\pi}$. Refinements of $\rho_{\pi}$ that can be obtained this way are called accessible.

2.6. The weight space. We define a weight space $W_{\mathbb{Q}_{p}}:=\operatorname{Hom}_{\text {rig }}\left(\mathbb{Z}_{p}^{*}, G_{m}\right)^{2}$ of dimension 2 , and embed $\mathbb{Z}^{2}$ as a Zariski-dense subspace in $W_{\mathbb{Q}_{p}}\left(\mathbb{Q}_{p}\right)$ in a natural way.

2.7. Representations that are cohomological in degree 1 . Let $\chi_{0}$ be a Grossencharacter of $E$ such that for all $z \in \mathbb{A}_{E}, \chi_{0}(z \bar{z})=1$ and such that for $z \in E \otimes \mathbb{R}=\mathbb{C}$, $\left(\chi_{0}\right)_{\infty}(z)=z^{k} /(z \bar{z})^{k / 2}$, where $k$ is an odd integer different from \pm 1 . (The oddness of $k$ is equivalent to the property that $\chi_{0}$ does not come from a Hecke-character of $\mathrm{U}(1)$.)

The complete $L$-function $L\left(\chi_{0}, s\right)$ satisfies a functional equation (see [Ta, 3.6.8 and 3.6.1])

$$
L\left(\chi_{0}, 1-s\right)=\epsilon\left(\chi_{0}, s\right) L\left(\chi_{0}, s\right), \quad \epsilon\left(\chi_{0}, 1 / 2\right)= \pm 1 .
$$

For every place $v$ of $\mathbb{Q}$, we can define (following Rogawski) an irreducible admissible representation $\pi^{n}\left(\chi_{0}\right)_{v}$ of $G\left(\mathbb{Q}_{v}\right)$ which is non tempered (and unramified if $v$ is finite, unramified in $E$, and $\left(\chi_{0}\right)_{v}$ is unramified) as follows (see [BC1, $\S 4.2$ and $\left.\S 4.3\right]$ for more details):

If $v$ is split in $E$, the choice of a place $w$ above $v$ determines an isomorphism $G\left(\mathbb{Q}_{v}\right) \simeq \mathrm{GL}_{3}\left(\mathbb{Q}_{v}\right)$, well defined up to conjugacy, and if $P=M N$ is the standard parabolic of type $(2,1)$ of $\mathrm{GL}_{3}\left(\mathbb{Q}_{v}\right)$ (so $\left.M=\mathrm{GL}_{2}\left(\mathbb{Q}_{v}\right) \times \mathrm{GL}_{1}\left(\mathbb{Q}_{v}\right)\right), \pi^{n}\left(\chi_{0}\right)_{v}$ is the normalized parabolic induction of the character $M \rightarrow \mathbb{C}^{*},(x, y) \mapsto \chi_{0, v}(\operatorname{det}(x))|\operatorname{det}(x)|_{v}^{1 / 2}$.

If $v$ is a inert or ramified in $E$ (including the infinite place), and $w$ the unique place of $E$ above $v$ then in a suitable basis the maximal torus of $G\left(\mathbb{Q}_{v}\right)$ is

$$
\left\{\operatorname{diag}\left(\alpha, \beta, c(\alpha)^{-1}\right), \quad \alpha, \beta \in E_{w}^{*}, \beta c(\beta)=1\right\},
$$

and $\pi^{n}\left(\chi_{0}\right)_{v}$ is the normalized parabolic induction of the character

$$
\left.\operatorname{diag}\left(\alpha, \beta, c(\alpha)^{-1}\right) \mapsto \chi_{0, w}(\alpha)|\alpha|_{w}^{1 / 2}\right) .
$$

In addition, for $v$ inert or ramified, Rogawski constructs by difficult trace formula considerations a square-integrable (even supercuspidal when $v$ is finite) representation $\pi^{s}\left(\chi_{0}\right)_{v}$ of $G\left(\mathbb{Q}_{v}\right)$. Rogawaki has also defined local and global $A$-packets for the group $G$ in accordance of Arthur's formalism. The representations $\pi^{n}\left(\chi_{0}\right)_{v}$ belongs to only one local $A$-packet; it is a singleton when $v$ is split in $E$, and the pair $\left\{\pi^{n}\left(\chi_{0}\right)_{v}, \pi^{s}\left(\chi_{0}\right)_{v}\right\}$ when $v$ is ramified or inert in $E$ (including the non-archimedean place of $\mathbb{Q})$. A representation of the global corresponding $A$-packet $\Pi\left(\chi_{0}\right)$ is an irreducible admissible representation of $G\left(\mathbb{A}_{\mathbb{Q}}\right)$ of the form $\pi=\otimes_{v} \pi_{v}$, where $\pi_{v}=\pi^{n}\left(\chi_{0}\right)_{v}$ for almost all $v$, and $\pi_{v}=\pi^{s}\left(\chi_{0}\right)_{v}$ otherwise. Let $n(\pi)$ be the number of places $v$ of $\mathbb{Q}$ such that $\pi_{v}=\pi^{s}\left(\chi_{0}\right)_{v}$. 
Fact 1 (Rogawski). (i) Assume that $\chi_{0}, \pi$ are as above, that $\pi_{\infty}=\pi^{n}\left(\chi_{0}\right)_{\infty}$, and that $(-1)^{n(\pi)}=\epsilon\left(\chi_{0}, 1 / 2\right)$. Then $\pi$ is discrete, and cohomological in degree 1 (and 3). Moreover $\pi$ is cuspidal, except in the case where $n(\pi)=0$ and $L\left(1 / 2, \chi_{0}\right) \neq 0$, where $\pi$ is residual (that is, discrete non cuspidal)

(ii) Conversely, any discrete automorphic representation of $G$ that is cohomological in degree 1 is of the form described in (i).

Reference for this fact are: $[\operatorname{Rog} 2]$ for the definition of $\pi^{n}, \pi^{s}$ and the existence assertion; [Rog3] for the cuspidality assertion, and [Rog1, 4.4] for the cohomological assertions of (i), and (ii). (For (ii), see also [MR, Prop 3] together with the classification recalled in $[\mathrm{BC} 1, \S 3])$.

The character $\chi(z)=\chi_{0}(z)(z \bar{z})^{1 / 2}$ is algebraic. We still denote by $\chi$ its $p$-adic realization as a character $\Gamma_{E} \rightarrow \overline{\mathbb{Q}}_{p}$. We have $\chi^{\perp}=\chi(1)$.

Fact 2 (Rogawski). If $\pi$ is as in the above fact, then $\rho_{\pi}$ is the sum of three characters $\chi \oplus 1 \oplus \chi^{\perp}$, and the weights $k_{0}<k_{1}<k_{2}$ of $\rho_{\pi}$ satisfy either $k_{1}-k_{0}=1$ or $k_{2}-k_{1}=1$

Indeed, the description of $\rho_{\pi}$ follows directly form the description of $\pi^{n}$ in [Rog2] (see also [BC1, §3.2.3]), and it follows that (if $v$ is well chosen) the weights of $\rho_{\pi}$ are in some order $0,(k-1) / 2,(k+1) / 2$, which implies that $k_{2}-k_{1}=1$ if $k>1$, and $k_{1}-k_{0}=1$ if $k<-1$.

Remark 1. It is easy to see that if the level $K^{p}$ is small enough, then for all triples of integers $\left(k_{0}, k_{1}, k_{2}\right)$ that satisfies either $k_{1}-k_{0}=1$, or $k_{2}-k_{0}=1$, there are exists representations $\pi$ as in Fact 1 with weights $\left(k_{0}, k_{1}, k_{2}\right)$. The Zariski closure of those weights in the three dimensional weight space obviously has dimension 2 , or if we fix one of the weight, for example the first one $k_{0}$ to 0 , has dimension 1 . So it is natural to expect that there exist one-dimensional non-trivial $p$-adic families interpolating forms that are cohomological in degree 1 . Theorem 1 shows that actually it is not always the case.

Let $\pi$ be as in Fact 1 . Assume in addition that $\pi_{p}$ is unramified. By Fact $2, \rho_{\pi}$ is the sum of three characters, so the weak admissibility conditions applied to each of them gives that the set of weights $\left\{k_{0}, k_{1}, k_{2}\right\}$ is equal to the set of slopes $\left\{s_{0}, s_{1}, s_{1}\right\}$ of $\rho_{\pi}$. Thus, a refinement of $\rho_{\pi}$ is an ordering of the weight $\left\{k_{0}, k_{1}, k_{2}\right\}$.

Fact 3. If $\pi_{p}$ is unramified, then $\pi_{p}^{I_{p}}=\pi^{n}\left(\chi_{p}\right)^{I_{p}}$ has dimension 3 , and $\pi$ has three possible refinements. Assume to fix ideas that $k_{2}-k_{1}=1$ (the other case being symmetric). The corresponding accessible refinements of $\rho_{\pi}$ are (a) $\left(k_{0}, k_{2}, k_{1}\right) ;(b)$ $\left(k_{2}, k_{0}, k_{1}\right)$, and (c) $\left(k_{2}, k_{1}, k_{0}\right)$

This follows from [BC1, Remark 5.2.4].

Remark 2. - The ordinary refinement would be $\left(k_{0}, k_{1}, k_{2}\right)$. It is not accessible in the present situation. In other words, we are not in a case where Hida's work allows us to construct $p$-adic families through $\pi$.

- The refinement (b), that is, $\left(k_{2}, k_{0}, k_{1}\right)$ is anti-ordinary in the sense of [BC2, $\S 2.4]$. It is the refinement used in the paper [BC1].

- The refinement (a) is what Urban and Skinner call semi-ordinary. It is analog to the one they use in their paper [SU2]. 


\section{Rigidity of cohomological in degree 1 forms.}

3.1. Definition of a $p$-adic family of forms that are cohomological in degree $n$. For our purpose, we will adopt the following, rather weak, notion of $p$-adic family. Let $K$ be as in $\S 2.3$

Definition 1. Let $n$ be an integer. By a $p$-adic family interpolating discrete automorphic representations of level $K$ and cohomological of degree $n$ for $\mathrm{U}(2,1)$, or for short a p-adic family, we mean the data of

- A rigid analytic space $X$ over $\mathbb{Q}_{p}$ that is reduced and separated.

- An analytic map $\kappa=\left(\kappa_{1}, \kappa_{2}\right): X \rightarrow W_{\mathbb{Q}_{p}}$ over $\mathbb{Q}_{p}$.

- A ring homomorphism $\psi: \mathcal{H} \rightarrow \mathcal{O}(X)^{\text {rig. }}$

- A subset $Z \in X\left(\overline{\mathbb{Q}}_{p}\right)$ that is Zariski-dense and accumulates at every of its points.

such that

(a) For every $z \in Z$, the character $\psi_{z}: \mathcal{H} \rightarrow \mathcal{O}(X)^{\text {rig }} \stackrel{\text { eval. at }}{\rightarrow}^{\overline{\mathbb{Q}}_{p}}$ is of the form $\psi_{\pi, \mathcal{R}}$ where $\pi$ is of level $K$ and cohomological of degree $n$.

(b) If $z \in Z$, and $\psi_{z}=\psi_{\pi, \mathcal{R}}$, then the Hodge-Tate weights of $\rho_{\pi}$ are the natural integers $0, \kappa_{1}(z), \kappa_{2}(z)$, and we have $0 \leq \kappa_{1}(z) \leq \kappa_{2}(z)$.

(c) The map $Z \rightarrow \operatorname{Hom}\left(\mathcal{H}, \overline{\mathbb{Q}}_{p}\right), z \mapsto \psi_{z}$ is injective.

It is expected that eigenvarieties, if they exist, satisfy those properties, and many more: see [BC1, Definition 7.2.5 and Theorem 7.3.1] or [E, Theorem 0.7].

Remark 3. The condition (c) is a condition of non-triviality, though very weak. It ensures that the family is not the constant family, for example.

3.2. Rigidity. We shall say that a given refined automorphic representation belongs to the family $X$ if there is a $z \in Z$ such that $\psi_{z}=\psi_{\pi, \mathcal{R}}$.

Theorem 1. Let $X$ be an irreducible p-adic family as in definition 1 of cohomological degree $n=1$. Suppose that a refined discrete representation $\pi$ with a refinement $\mathcal{R}$ of type (b) or (c) of Fact 3 belongs to $X$. Then $X$ is a point.

Proposition 1. Let $X$ be a family as in the definition 1. There exists a pseudocharacter $T: \Gamma_{E} \rightarrow \mathcal{O}(X)^{\text {rig }}$ and three invertible analytic functions $F_{0}, F_{1}, F_{2}$ on $X$ such that for every $z \in Z$, such that if $\psi_{z}=\psi_{\pi, \mathcal{R}}$, and $\rho_{z}$ is the semi-simple representation of trace $T_{z}:=$ eval $_{z} \circ T$, then $\rho_{z} \simeq \rho_{\pi}$, and the eigenvalues or $\rho_{\pi}$ are $F_{0}(z), F_{1}(z) p^{\kappa_{1}(z)}, F_{2}(z) p^{\kappa_{2}(z)}$, given in the order defined by the refinement $\mathcal{R}$.

Proof - See $[\mathrm{BC} 1, \S 7]$. The functions $F_{0}, F_{1}, F_{2}$ are defined as the images by $\psi$ of three suitable elements in the Atkin-Lehner algebra $\mathcal{A}_{p}$.

Let us now assume, by contradiction, that $\operatorname{dim}(X)>0$. By hypothesis, every $z$ in $Z$ corresponds to a $\pi$ that is cohomological in degree 1 , so the weights of $\pi$, that is (by (b) of the definition of a family) $0 \leq \kappa_{1}(z) \leq \kappa_{2}(z)$ satisfy, by Fact 2, either $\kappa_{1}(z)=1$ or $\kappa_{2}(z)-\kappa_{1}(z)=1$. Since $X$ is irreducible, and $Z$ Zariski-dense, one of those two equalities has to hold for all $z \in Z$ (and even over $X$ ). By symmetry, we may and do assume that we have for all $z \in Z$,

$$
\kappa_{2}(z)-\kappa_{1}(z)=1 \text {. }
$$


Now, by hypothesis, there exists a $z_{0}$ in $Z$ such that the refinement is not the refinement (a). That is to say, the refinement at $z_{0}$ is either $\left(\kappa_{2}\left(z_{0}\right), \kappa_{1}\left(z_{0}\right), 0\right)$ or $\left(\kappa_{2}\left(z_{0}\right), 0, \kappa_{1}\left(z_{0}\right)\right)$. Using Prop. 1 we see that in both cases $v_{p}\left(F_{0}\left(z_{0}\right)\right)=\kappa_{2}\left(z_{0}\right)$.

Let $U$ be an affinoid neighborhood of $z_{0}$ on which the function $v_{p}\left(F_{0}\right)$ is constant. By hypothesis $Z \cap U$ is infinite. Since there is only a finite number of automorphic representations with a fixed level and fixed weight, there exists $z \in Z \cap U$ such that $\kappa(z) \neq \kappa\left(z_{0}\right)$. This implies $\kappa_{2}(z) \neq \kappa_{2}\left(z_{0}\right)$, since $\kappa_{1}=\kappa_{2}-1$ everywhere. But by Prop. 1 applied to $z$, we have $\kappa_{2}(z)=v_{p}\left(F_{2}(z)\right)=v_{p}\left(F_{2}\left(z_{0}\right)\right)=\kappa_{2}\left(z_{0}\right)$, a contradiction. This proves Theorem 1 .

Remark 4. Starting with a 1-dimensional deformation $\chi_{x}$ (for $x$ a parameter in a rigid analytic space $X$ ) of the Hecke character $\chi$, it is very easy to construct a one dimensional family as above of representation that are cohomological of degree 1 , refined with a refinement of type (a). This may be done "by hand", defining explicitly the function $\psi(h), h \in \mathcal{H}$, with the guidance that $\rho_{x}=1 \oplus \chi_{x} \oplus \chi_{x}^{\perp}$. We leave the details to the reader.

Remark 5. For higher rank unitary groups over $\mathbb{Q}$ (of signature $(n-1,1)$ at infinity), it should be possible to exhibit similar examples of rigidity in cohomological dimension 1, using instead of Fact 2 the main theorem of [MR], but we have not written down the details.

\section{Another rigidity result}

In this section, we give another rigidity result, which holds for a much smaller class of representations, but for a more general notion of family, where we do not assume the classical representations to have a fixed cohomological degree.

4.1. A special non-tempered refined representation. Let $\chi_{0}$ be Hecke character of $E$ as in $\S 2.7$, and assume that

$$
L\left(\chi_{0}, 1 / 2\right) \neq 0 .
$$

In particular, $\epsilon\left(\chi_{0}, 1 / 2\right)=1$, and by Fact 1 there exists a discrete automorphic representation $\pi^{n}\left(\chi_{0}\right)=\otimes_{v} \pi^{n}\left(\chi_{0}\right)_{v}$ for $G=\mathrm{U}(2,1)$. Let $\mathcal{R}$ be the refinement (b) of $\pi^{n}\left(\chi_{0}\right)_{p}$ (see Fact 3$)$.

4.2. Definition of a family. We fix a subgroup $K$ of $\mathrm{U}(2,1)\left(A_{\mathbb{Q}}\right)$ and a set $S$ of bad primes as in 2.3. We set $S^{p}=S-\{p\}$.

Since the argument we shall give is arithmetic, we shall need to control the ramification at every places so we must have a condition at primes of $S^{p}$. This can be done by imposing some types (in the sense of Bushnell and Kutzko) at those primes as in [BC1] or more conveniently, by using the notion of Non Monodromic Principal Series as in $[\mathrm{BC} 2, \S 6.6]$. We choose the second way. Morally, a Non Monodromic Principal Series is a principal series whose base change to $\mathrm{GL}_{n}$ corresponds by local Langlands to a representation of the Weil-Deligne group without monodromy, that is with $N=0$. For the precise definition, we refer the reader to [BC2, Def. 6.6.5].

Definition 2. By a strong p-adic family interpolating discrete cohomological automorphic representations of level $K$ for $\mathrm{U}(2,1)$, or for short a strong p-adic family, we mean the data of 
- A rigid analytic space $X$ over $\mathbb{Q}_{p}$ that is reduced and separated.

- An analytic map $\kappa=\left(\kappa_{1}, \kappa_{2}\right): X \rightarrow W_{\mathbb{Q}_{p}}$ over $\mathbb{Q}_{p}$.

- A ring homomorphism $\psi: \mathcal{H} \rightarrow \mathcal{O}(X)^{\text {rig. }}$

- A subset $Z \in X\left(\overline{\mathbb{Q}}_{p}\right)$ that is Zariski-dense and accumulates at every of its points.

such that

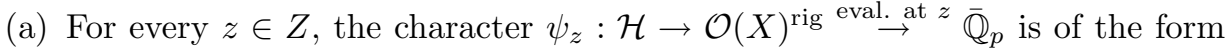
$\psi_{\pi, \mathcal{R}}$, where $\pi$ is of level $K$, cohomological of some degree, and $\pi_{l}$ is a Non Monodromic Principal Series (see [BC2, Def 6.6.5]) for all $l \in S^{p}$.

(b) If $z \in Z$, and $\psi_{z}=\psi_{\pi, \mathcal{R}}$, then the Hodge-Tate weights of $\rho_{\pi}$ are the natural integers $0, \kappa_{1}(z), \kappa_{2}(z)$, and we have $0 \leq \kappa_{1}(z) \leq \kappa_{2}(z)$.

(c) The map $Z \rightarrow \operatorname{Hom}\left(\mathcal{H}, \overline{\mathbb{Q}}_{p}\right), z \mapsto \psi_{z}$ is injective.

4.3. The Kisin property. The proof of Prop. 1 works without any change for a family $X$ as above. We keep its notations. For $z \in Z$, we denote by $\mathcal{O}_{z}$ the rigid analytic local field of $X$ at $z$.

Definition 3. A realization of $T$ over $\mathcal{O}_{z}$ is any torsion-free finite module $M$ over $\mathcal{O}_{z}$ with a continuous action of $\Gamma_{E}$, such that for all $g \in \Gamma_{E}, \operatorname{tr}\left(g \mid M \otimes \operatorname{Frac}\left(\mathcal{O}_{z}\right)\right)$ is in $\mathcal{O}_{z}$ and is equal to the germ of $T(g)$ at $z$.

Definition 4 . We shall say that the family $X$ satisfies the Kisin property at $z \in Z$ if for any realization $M$ of $T$ over $\mathcal{O}_{z}$ we have, if $\operatorname{dim} D_{\text {crys }}\left(M_{z}^{\text {ss }}\right)^{\phi=F_{0}}(z) \leq 1$, then $\operatorname{dim} D_{\text {crys }}\left(M_{z}\right)^{\phi=F_{0}(z)}=1$.

We say that the dual Kisin property holds if the same implication holds with $T$ replaced by the dual character $T\left(g^{-1}\right)$ and $F_{0}(z)$ by $p^{-\kappa_{2}(z)} F_{2}(z)$.

We say that the strong Kisin property holds if both the Kisin and the dual Kisin properties hold.

It is expected that eigenvarieties, and most of the naturally constructed families satisfies these properties. For the case of the eigencurve, this is essentially proved in [Ki], and for the eigenvarieties on unitary groups, and more generally families with a sufficiently dense set of "classical" points, in [BC2, §3.4].

Remark 6. The reason for which I state the Kisin property as a definition rather than as a theorem is that I do not want to presume what are the minimal conditions on a family that implies it. As I have just said, this property has been proved in [BC2] under some assumptions on the set $Z$, and a similar property is stated under a different assertion on $Z$ in [SU, Prop. 4.2.2(i)], without proof - the reference given there to [Ki] must be a joke. Anyway, since we want to prove the most general possible rigidity result in its line of reasoning, it is better to assume directly the Kisin property we need rather than any property on the family that implies it.

Another remark is that the formulation of the Kisin property given above, for general torsion free (as opposite to free, or locally free) realizations, may seem weird and complicated. Indeed, this formulation has been chosen merely for convenience. Let me explain the issue at stake. In general, it is not possible to choose in the neighborhood of a reducible point $z_{0}$ a realization of the pseudo-character $T$ that is free (see the discussion in [BC2, Chapter 1] and [B, Remark 9]). However, as far as we only want a Kisin's property on the representation at a closed point, we may in 
practice perform some surgery on $X$ to get a free module. One way to do so, simple though unestehtic, is to replace $X$ by the normalization on a suitable curve in $X$ through $z_{0}$. (This is the way used in [BC1], and later in [SU2]). Another way is to replace $X$ by a suitable blow-up (a normalization may not be enough, as shown by the example developed in $[\mathrm{B}]$, since the normalization of the family defined in $[\mathrm{B}, \S 5]$ still satisfies the properties (i) to (ix) loc. cit.), idea which is used in [BC2, Chapter 3] (and before, though for a different purpose, in [Ki]). Now, those surgical operations work for real-world families, but in an abstract setting like ours (or the one of [SU]), one would need a definition of family which is stable by those operations. This would lead to unnecessarily restrictive and unesthetic assumptions in the definition of a family. So we have chosen another way, namely a formulation of the Kisin property for general torsion-free modules, which is not much harder to prove in practice.

\subsection{Rigidity.}

Theorem 2. Let $X$ be an irreducible family as in Definition 2, and assume that $\left(\pi^{n}\left(\chi_{0}\right), \mathcal{R}\right)$ (defined in 4.1) belongs to $X$, and that $X$ satisfies the strong Kisin property at the corresponding point $z_{0}$. Then $X$ is a point.

Proof - Let $Z_{1}$ be the set of points of $Z$ which are cohomological of degree 1. If $Z_{1}$ is Zariski-dense, then replacing $Z$ by $Z_{1}$ we see that $X$ is a family in the sense of Definition 1 , and $X$ is a point by Theorem 1 . Therefore we can assume that $Z_{1}$ is not Zariski-dense.

Let $Z_{0}$ be the set of points such that $\psi_{z}=\psi_{\pi, \mathcal{R}}$ for $\pi$ a one dimensional representation. We have at those points $\rho_{z}=\mu_{1} \oplus \mu_{2} \oplus \mu_{3}$, with the three characters $\mu_{i}$ satisfying $\mu_{i}=\mu_{i}^{\perp}$. If $Z_{0}$ was Zariski-dense, the same property would hold for any $\rho_{z}, z \in Z$. But this is false for $\rho_{z_{0}} \simeq \chi \oplus 1 \oplus \chi^{\perp}$, since $\chi \neq \chi^{\perp}=\chi(1)$. Therefore, $Z_{0}$ is not Zariski-dense.

Hence we see that there is a Zariski-dense set of $z$ corresponding to points that are neither cohomological of degree 1, nor 1-dimensional. By Rogawski's classification (see $[\mathrm{BC} 1, \S 3.2 .3]$ ), those points have a $\rho_{z}$ that are either irreducible, or the sum of a character and a two-dimensional representation. Thus the dimension 3 pseudocharacter $T: \Gamma_{E} \rightarrow \mathcal{O}(X)^{\text {rig }}$ (see Prop 1) either is irreducible (that is not the sum of pseudocharacters of smaller dimension), or is the sum of a pseudocharacter of dimension 2 and a character.

For any pseudocharacter $T: \Gamma_{E} \rightarrow A$ where $A$ is a commutative ring, let us denote by $T^{\perp}$ the pseudocharacter $T^{\perp}(g)=T\left(c g^{-1} c\right)$ where $c$ is an element of order 2 in $\Gamma_{\mathbb{Q}}-\Gamma_{E}$. It is clear that the trace $T=\operatorname{tr} \rho$ for a representation $\rho$, then $T^{\perp}=$ $\operatorname{tr} \rho^{\perp}$. The application $T \mapsto T^{\perp}$ is an involution preserving dimension on the set of pseudocharacters of $\Gamma_{E}$ over $A$. Going back to our pseudocharacter $T: \Gamma_{E} \rightarrow \mathcal{O}(X)^{\mathrm{rig}}$ defined in Prop 1, we see at once that $T=T^{\perp}$, since $T_{z}=T_{z}^{\perp}$ for all $z \in Z$.

This being secured, we treat the two cases ( $T$ irreducible, or the sum of a pseudocharacter of dimension 2 and 1) separately, leading in both cases to a contradiction (if $\operatorname{dim} X>0$ ).

If $T$ is the sum of a pseudocharacter $T^{\prime}$ of dimension 2 and of a character (the simplest case), then by dimensions we have $T^{\prime}=T^{\prime \perp}$ and in particular $T_{z_{0}}^{\prime}=$ $T_{z_{0}}^{\prime \perp}$. On the other hand, $T_{z_{0}}^{\prime}$ being a sub-pseudochatacter of $T_{z_{0}}=\chi \oplus 1 \oplus \chi^{\perp}$, 
is the sum of two among the three characters $\chi, \chi^{\perp}, 1$. The only possibility is that $T_{z_{0}}^{\prime}=\chi \oplus \chi^{\perp}$.

From this, by an application of the generalized Ribet's lemma of [BC2], we can construct a non trivial extension $U$ of $\chi$ by $\chi^{\perp}$ (which appears as a sub-quotient of some realization $M$ of $T$ over $\mathcal{O}_{z_{0}}$, such that $D_{\text {crys }}\left(M_{z_{0}}^{\text {ss }}\right)^{\phi=F_{0}}\left(z_{0}\right)$ of dimension $1-$ see [BC2, Theorem 1.5.6] for the proof) that moreover satisfies $U \simeq U^{\perp}$ (see [BC2, $\S 1.9])$. From the Kisin properties at $z_{0}, U$ is crystalline at $v$. Since $U \simeq U^{\perp}$ it is also crystalline at $\bar{v}$. The twist $U \chi^{-1}$ is an extension of 1 by $\overline{\mathbb{Q}}_{p}(1)$ which is crystalline at $v$ and $\bar{v}$, and unramified everywhere else by the Non Monodromic Principal Series assumption (see [BC2, Prop 8.2.10]). As is well known, there is no such extension in the category of $\Gamma_{E}$-representation, $E$ a quadratic imaginary field (see e.g. [BC2, Prop. 5.2.2]). This is a contradiction.

If $T$ is irreducible then we can proceed exactly as in [BC2, Chapter VIII] or in $[\mathrm{BC} 1, \S 9]$ to construct a non-trivial element element in $H_{f}^{1}(E, \chi)$. But since $L\left(\chi_{0}, 1 / 2\right) \neq 0$ by assumption, that is $L(\chi, 0)=L\left(\chi^{\perp}(1), 0\right) \neq 0$, Bloch-Kato's conjecture predicts that $H_{f}^{1}(E, \chi)=0$. This prediction is a consequence of a theorem of Rubin $([\mathrm{Ru} 2])$ and also follows from Kato $([\mathrm{Ka}])$. Indeed, $H_{f}^{1}(E, \chi)=H_{f}^{1}\left(\mathbb{Q}, \operatorname{Ind}_{E}^{\mathbb{Q}} \chi\right)$ by Shapiro's lemma, and since $\operatorname{Ind}_{E}^{\mathbb{Q}} \chi$ is up to a Tate twist the Galois representation attached to a (CM) modular form, the upper bound on the dimension of Selmer group of $\operatorname{Ind}_{E}^{\mathbb{Q}} \chi$ predicted by the Bloch-Kato conjecture is precisely the main theorem of $[\mathrm{Ka}]$. This is the contradiction we were looking for.

For the commodity of the reader, we sketch the argument leading to the construction of an element of $H_{f}^{1}(E, \chi)$. The long technical details are given twice, in slightly different settings in [BC2, Chapter VIII] or in [BC1, §9], and I do not think that it might be useful to give them a third time here.

Since $T$ is irreducible, while $T_{z_{0}}$ is reducible (the sum of three characters), we are in position to apply a suitable generalization of Ribet's lemma. We have two possible paths, essentially equivalent but technically different (and both relatively perilous). We first can choose a suitable line through $z_{0}$ in $X$, normalize it, and reduce to a case where $T$ is the trace of a true representation over a d.v.r, generically irreducible but residually isomorphic (up to semi-simplification) to $1 \oplus \chi \oplus \chi^{\perp}$. We can then apply Ribet's generalized lemma for representations (cf. [BG]). Or better, we can use the theory of reducibility ideal and the Ribet's Lemma for pseudocharacter of [BC2, Chapter I]: the assumption that $T$ is irreducible precisely says that the reducibility ideal is non zero, which in the context of [BC2] is a difficult result [BC2, Lemma 8.3.3]. In both cases, the variant of Ribet's lemma implies the existence of either a non-trivial extension of $\chi^{\perp}$ by 1 , or of a non-trivial extension of $\chi$ by $\chi^{\perp}$. Moreover, it can be proved (that the most technical point, for which the arguments of [BC2, Chapter VIII] work with the only changes that [BC2, Lemma 8.3.3] is true by assumption in our context (so one can disregard its proof), and that we need to replace the references to Kisin's lemma there by our assumption that $T$ satisfies the strong Kisin properties) that those extensions satisfies after twisting by $\chi^{-1}$ the Bloch-Kato condition at every places of $E$ (see [BC2, Chapter VIII] for details). We have already seen that such an extension of $\chi$ by $\chi^{\perp}$ does not exist. Therefore, a non-trivial, Bloch-Kato, extension of $\chi^{\perp}$ by 1 , that is (by twisting or applying $\perp$ ), a non-trivial, Bloch-Kato, extension of 1 by $\chi$ has to exist, and defines a non trivial element in $H_{f}^{1}(E, \chi)$. 
Remark 7. It might be interesting to compare the proof of the theorem above with the family-theoretic proof of the non-overconvergence of the $p$-adic modular form $E_{2}$. This proof, which seems to have been discovered independently by several authors can be found in the appendix of [C1] and goes roughly as follows: if $E_{2}$ was overconvergent, then choosing a refinement of it, one could put it by Coleman theory into a non-trivial family. If we pick the non-ordinary refinement, we see that this family has to be generically irreducible and leads by Ribet's lemma to a non-trivial extension from $\mathbb{Q}_{p}$ by $\mathbb{Q}_{p}(1)$ in the category of $\Gamma_{\mathbb{Q}}$-representations, which actually defines a non-zero element of $H_{f}^{1}\left(\Gamma_{\mathbb{Q}}, \mathbb{Q}_{p}(1)\right)$. But the latter group is 0 , a contradiction.

The argument of the proof of the above theorem is similar in that it uses the same kind (though much harder) of Ribet-like constructions to finally get a non-existing element in some $H_{f}^{1}$. However, the conclusion is different. In the case of $E_{2}$, we know that if $E_{2}$ existed as an overconvergent modular form, the family through it would exist (by Coleman's work), so the only escape from the contradiction is the non overconvergence of $E_{2}$. In the case of $\pi_{n}\left(\chi_{0}\right)$, we know the existence of $\pi_{n}\left(\chi_{0}\right)$ (even as a classical automorphic form) by Rogawski's work, so the only possible conclusion is the non existence of the family.

Remark 8. It is also interesting to confront the above rigidity result with [SU, Theorem 3.2.1], which states the existence of two-dimensional families for unitary groups in some cases. As it stands, the rough sketch of proof of [SU, Theorem 3.2.1] which produces families of automorphic form through a given automorphic representation $\pi$ applies smoothly to our representation $\pi^{n}\left(\chi_{0}\right)$, leading to a contradiction with the rigidity theorem 2. Unfortunately, three years ${ }^{3}$ after the appearance in print of [SU], the authors have not released their proof yet, so one can only try to guess what specific difference between our situation and theirs will solve the contradiction. Since our notions of family are essentially the same (both are families with a dense set of cohomological points, see [SU, Def. 2.3.1]), this difference is certainly an assumption on the automorphic representation needed to make work their proof of the existence of a non-trivial family, assumption that is satisfied in their case but not for $\pi^{n}\left(\chi_{0}\right)$. This assumption might be the $p$-adic cuspidality of $\pi$, but I am not sure what part it would play in the proof (this notion in not mentioned in their sketch of proof, though it is elsewhere in [SU]). It might also be a condition on $\pi_{\infty}$ needed to apply the Kisin-Lai method to $\pi$, such has being holomorphic, but since $\pi^{n}\left(\chi_{0}\right)_{\infty}$ has cohomology in degree 1 , this form may also be represented by a section of some holomorphic automorphic bundle on the Shimura variety, and we would need the details on their generalization of the Kisin-Lai method for $\mathrm{U}(2,1)$ to see what actually happens.

\section{References}

[APS] A. Ash, D. Pollack, G. Stevens, Rigidity of p-adic cohomology classes of congruence subgroups of $G L(N, \mathbb{Z})$, preprint, available on http://math.bu.edu/people/ghs/research.d.

[B] J. Bellaïche, Non smooth classical points on eigenvarieties, Duke Math. Journal.

[BC1] J. Bellaïche \& G. Chenevier, Formes non tempérées pour U(3) et conjectures de Bloch-Kato, Ann. Sci. E.N.S. 37 (2006), no. 4, 611-662.

\footnotetext{
$3_{\text {four years (added in proofs) }}$
} 
[BC2] J. Bellaïche \& G. Chenevier, p-adic families of Galois representations and higher rank Selmer groups, Astérisque 324, S.M.F. (or ArXiV math.NT/0602340.)

[BG] J. Bellaïche \& P. Graftieaux, Représentations sur un anneau de valuation discrète complet, Math. Ann. 334 (2006), 465-488.

[BR1] D. Blasius et J. Rogawski, Tate class and arithmetic quotient of two-ball in The Zeta Function of Picard Modular Surfaces, CRM, 421-443.

[C1] G. Chenevier, Familles p-adiques de formes automorphes et applications aux conjectures de Bloch-Kato, available on http://www.math.polytechnique.fr/ chenevier/pub.html

[C] G. Chenevier, Familles p-adiques de formes automorphes pour $G L(n)$, Journal für die reine und angewandte Mathematik 570 (2004), 143-217.

[Corv] Automorphic forms, representations and L-functions, Corvallis conference, Proceedings of Symposia in Pure Mathematics 33 (1977).

[E] M. Emerton, On the interpolation of systems of eigenvalues attached to automorphic Hecke eigenforms, Invent. Math. 164 no.1 (2006), 1-84.

[Ka] K. Kato, p-adic Hodge theory and values of zeta functions of modular forms, Astérisque 295 (2004), p. 117-290.

[Ki] M. Kisin, Overconvergent modular forms and the Fontaine-Mazur conjecture, Inv. Math. 153(2) (2003), 373-454.

[Rog1] J. Rogawski, Analytic expression for the number of points mod p, [ZFPMS], 65-109

[Rog2] J. Rogawski, The multiplicity formula for A-packets, [ZFPMS], 395-419

[Rog3] J. Rogawski, Automorphic representations of unitary groups in three variables, Annals of math. studies 123, Princeton University Press (1990)

[SU] C. Skinner \& E. Urban, Vanishing of L-functions and ranks of Selmer groups, in International Congress of Mathematicians, Vol. II, Eur. Math. Soc., Zurich, 2006, p. 473-500.

[SU2] C. Skinner \& E. Urban, Sur les déformations p-adiques des formes de Saito-Kurokawa, Journal of the Institute of Mathematics of Jussieu, 5 (2006), 629-698

[Ta] J. Tate, Number theoretic background, in [Corv] Part 2, 3-26.

[Ru2] K. Rubin, The "main conjectures" of Iwasawa theory for imaginary quadratic fields, Inventiones math. 103(1) (1991), 25-68.

[MR] V. K. Murty \& Dinakar Ramakrishna, The Albanese of unitary Shimura variety, [ZFPMS], 445-464

[ZFPMS] Ed. R. Langlands \& D. Ramakhrisna, The Zeta functions of Picard modular surfaces, Publications C.R.M., Montréal (1992).

Mathematics Department, Brandeis University, 415 South Street, Waltham, MA 024549110, U.S.A

E-mail address: jbellaic@brandeis.edu 